\title{
Expiratory Flow Accelerator (EFA) technique on mucus hypersecretion of COPD patients with reduced cough efficiency after a severe exacerbation
}

\author{
Elisabetta Zampogna ${ }^{1}$, Ernesto Crisafulli ${ }^{2,3}$, Michele D'Andria ${ }^{4}$, Cristina Gregorini ${ }^{4}$, Giorgio Bellelli ${ }^{1}$, Etienne Lucini ${ }^{1}$, Silvia Faverzani ${ }^{5}$, \\ Dina Visca ${ }^{6}$, Antonio Spanevello ${ }^{6}$, Nicola Schiavone ${ }^{7}$, Alfredo Chetta ${ }^{5}$ and Andrea Zanini ${ }^{7}$ \\ ${ }^{1}$ Division of Pulmonary Rehabilitation, Istituti Clinici Scientifici Maugeri, IRCCS, Tradate, Italy \\ ${ }^{2}$ Department of Medicine, Respiratory Medicine Unit, University of Verona and Azienda Ospedaliera Universitaria Integrata of Verona, Verona, Italy \\ ${ }^{3}$ Department of Medicine, Section of Internal Medicine, University of Verona and Azienda Ospedaliera Universitaria Integrata of Verona, Verona, Italy \\ ${ }^{4}$ Division of General Medicine, Ospedale Malcantonese, Castelrotto, Switzerland \\ ${ }^{5}$ Department of Medicine and Surgery, Respiratory Disease and Lung Function Unit, University of Parma, Parma, Italy \\ ${ }^{6}$ Department of Medicine and Surgery, Respiratory Diseases, University of Insubria, Varese-Como, Italy \\ ${ }^{7}$ Pulmonary Rehabilitation, Clinic of Rehabilitation, Ente Ospedaliero Cantonale, Novaggio, Switzerland
}

\begin{abstract}
Background: Mucus hypersecretion has a negative impact in chronic obstructive pulmonary disease (COPD). Expiratory Flow Accelerator (EFA) technique removes secretions noninvasively by accelerating expiratory flow. The aim of our study was to compare EFA efficacy with the non-oscillatory positive expiratory pressure (PEP) bottle in airways clearing in COPD with hypersecretion and reduced cough efficiency after severe exacerbation.

Methods: In an exploratory prospective, randomized, open-label study, we analyzed COPD patients with severe airflow obstruction (forced expiratory volume at $1^{\text {st }}$ second-FEV ${ }_{1}<50 \%$ ), mucus hypersecretion (sputum volume $>30 \mathrm{ml} /$ die) and reduced cough efficiency (peak cough expiratory flow-PCEF $<3001 /$ min) referred to pulmonary rehabilitation after severe exacerbation. Comparison was made between group using EFA and group using PEP. Primary outcome was change in perceived bronchial encumbrance (visual analogue scale-VAS). Secondary outcomes were changes in peak expiratory flow (PEF), PCEF, lung volumes, arterial blood gases exchanges, maximum inspiratory and expiratory pressure (MIP and MEP), health status (COPD questionnaire score-CCQ) and impact of disease (COPD Assessment Test-CAT).
\end{abstract}

Results: Twenty seven patients completed the study (14 EFA, 13 PEP). VAS, CAT, and CCQ improved in both groups (p<0.05). A greater improvement in VAS was recorded in the EFA group. Static and dynamic volumes (residual volume-RV and forced vital capacity-FVC) improve in both groups while total lung capacity (TLC) and MIP improved in the EFA group. No significant changes were recorded for arterial blood gases, PEF and PCEF.

Conclusions: EFA technique can enhance airways clearing in these patients, improving lung volumes and inspiratory muscle strength.

\section{Introduction}

The course of chronic obstructive pulmonary disease (COPD) is often characterized by exacerbations (AECOPD) [1], that have a negative impact on the quality of life of patients, accelerate disease progression, and can result in hospital admissions and death [1,2]. Airway clearance techniques (ACT) involve application of physical forces to enhance removal of sputum from the airways [3]. Current evidence for the effects of airway clearance techniques in AECOPD is actually very little. Some positive effects have been reported in a Cochrane review [4], but these effects are small and are not supported by the results of a recent large trial [5]. While their efficiency is often debated, ACT remain widely prescribed and regularly used with patients who have AECOPD [6]. Moreover, many studies do not account for different COPD phenotypes, then this does not exclude a role for the ACT in carefully selected patients in whom excessive sputum production or sputum retention, due to reduced cough efficiency, are clinically important problems.
A new type of ACT, expiratory flow accelerator (EFA), has been introduced in recent years, which is employed in the new product Free Aspire Advanced (FA), in particular for home care use, and in the device Suction Free (SF), for hospital use. This technology accelerates the expiratory flow, promoting deep drainage and secretions without applying any pressure in the airways. These devices utilize Vaküm technology, which accelerates expiratory flow through the Venturi

${ }^{\star}$ Correspondence to: Ernesto Crisafulli, Department of Medicine, Respiratory Medicine Unit, University of Verona and Azienda Ospedaliera Universitaria Integrata of Verona, Address: P.le L.A. Scuro 10, 37134 Verona, Italy, Tel: 0458124263; E-mail: ecrisafulli265@gmail.com

Key words: chronic obstructive pulmonary disease, pulmonary rehabilitation, mucus hypersecretion, expiratory flow accelerator, positive expiratory pressure, bronchial encumbrance

Received: October 11, 2019; Accepted: October 27, 2019; Published: October 30, 2019 

exacerbation

effect from a special connector, and they are based on studies by Kim et al. $[7,8]$. The process happens only during the expiratory phase and is proportional to airflow on spontaneous breathing, according to the natural rhythm of the patient's respiratory function. The secretions slide along the layer of liquid lining the bronchial epithelium until they reach the glottis from where they are swallowed, therefore no respiratory effort is required for the elimination. No negative pressure is generated inside the lungs therefore there is no risk of airways collapse. EFA technique does not require an efficient cough and offers a gentle secretion removal solution.

Garuti et al. showed efficacy and safety of FA in reducing the impact of respiratory exacerbations in pediatric patients affected by cerebral palsy [9]. They found a reduction in home visits by primary care pediatrician, days spent in hospital, and days of antibiotic treatment for respiratory problems [9]. Similarly, Bertelli et al. demonstrated a positive effect of home FA treatment in children with cerebral palsy, in improving general health state and decreasing health care resources utilization, with a positive caregivers' perception [10]. In a case report on a 3-year-old girl with spinal muscular atrophy, Bertelli et al. showed that FA in patients is an effective device for the removal of bronchial secretions [11]. More recently, Patrizio et al. observed larger improvements in peak cough expiratory flow (PCEF) and maximal expiratory pressure (MEP) in severe stable COPD patients treated with FA in comparison with those treated with positive expiratory pressure (PEP) bottle [12].

The present study aimed to evaluate the efficacy of EFA in improving the bronchial clearance in COPD patients with hypersecretion and reduced cough efficiency after severe exacerbation, in comparison with PEP-bottle.

\section{Methods}

\section{Design of the study}

This is an exploratory prospective, randomized, open-label study designed to compare EFA technique and PEP-bottle in the clearance of bronchial secretions. We considered severe-to-very severe COPD patients (forced expiratory volume at $1^{\text {st }}$ second $\left[\mathrm{FEV}_{1}\right]<50 \%$ of predicted), having a chronic mucus hypersecretion and a reduced cough efficiency (see section on selection of patients). We assumed to enroll 40 patients (20 EFA and $20 \mathrm{PEP}$ ) but due to the difficulty to enroll this kind of patients, the study was closed earlier considering 34 patients (16 EFA and $18 \mathrm{PEP}$ ). The protocol was approved by the Ethics Committee on June 30, 2017, CE 2127 and recorded on the ClinicalTrial.gov website with identification number NCT02640430.

\section{Selection of patients}

We evaluated 96 COPD patients with severe airflow obstruction admitted from June 2017 and December 2018 for an inpatient pulmonary rehabilitation (PR) after severe exacerbation (CastelrottoSwitzerland and Istituti Clinici Scientifici Maugeri, Tradate-Italy). All subjects had a diagnosis of COPD according to Global Initiative for Chronic Obstructive Lung Disease criteria [1]. Contraindications for participation in the PR program included musculoskeletal disorders, malignant diseases, unstable cardiac condition, and lack of adherence to the program.

Chronic mucus hypersecretion was defined as a sputum volume production of greater than $30 \mathrm{ml} /$ day [13]. Chronic mucus hypersecretion was specifically related to COPD and patients with diagnosis of bronchiectasis were also excluded. All patients taking any active drugs to have a mucoregulatory action (i.e. aminophyllines, $\mathrm{N}$-acetyl-cysteine, erdostein) as well as systemic corticosteroid and antibiotics at selection time were also excluded. A reduced cough efficiency was confirmed by a PCEF between $150 \mathrm{l} / \mathrm{min}$ and $300 \mathrm{l} / \mathrm{min}$ [14]. All patients had a smoking history $\geq 10$ pack years and received regular treatment with inhaled bronchodilators and inhaled steroids according to current guidelines for their disease stage. Each patient signed an informed consent form.

\section{Rehabilitation program}

All patients included in the study followed a PR program, according to the international recommendations [15]. To be included in the study, patients had to perform at least 12 supervised sessions, up to a maximum of 15 sessions over a 3-week period. The PR program consisted of lower limb endurance and resistance training, as main component. All patients performed sessions of 30-40 minutes, using a treadmill or cycle-ergometer, depending on the clinically based choice of the physiotherapist, and on the subject's preference. Exercise intensity was based on the initial six-minute walking test (6MWT), and patients started their training at $70 \%-85 \%$ of the maximum heart rate (HR) achieved on the 6MWT [16]. Exercises were then adjusted based on patient tolerance (at least weekly) with the aim of achieving a Borg dyspnea score of 4-5 (moderate-severe). To optimize training load supplemental oxygen for patients with chronic respiratory failure and interval training for much compromised patients were adopted. Transcutaneous arterial oxygen saturation, arterial pressure, and HR were monitored during every exercise session. Resistance training is composed as 2 series of 10 repetitions of 6 exercises targeting all major muscle groups. Initial loads were equivalent to either 60\%-70\% of the one-repetition maximum or one that evoked fatigue after 8-12 repetitions. The exercise dosage was increased when the individual could perform the current workload for one or two repetitions over the requested number on two consecutive training sessions. Each session also included supervised upper limb training: patients used an arm ergometer or performed callisthenic exercises holding a light weight.

In addition, the study group received 10 daily supervised session (20 minutes twice a day) with the EFA device, and the control group 10 daily supervised sessions (20 minutes a day) with the PEP-bottle. For the study group we adopted the EFA device for hospitalized patients, named Suction Free".

Finally, each patient participated in educational activities, individually (at least one time) and in a group (at least three times), regarding self-management, airway clearance techniques, adherence to therapy, and nutritional support. The total daily time duration for all activities was 2-3 hours, and the entire program was conducted in the hospital.

\section{Measurements and outcomes}

At enrolment, patients' anthropometric and physiological characteristics and main diagnosis were recorded. Outcome measures were taken at study entry and after 12 days of treatment.

Primary outcome: Perceived symptom of bronchial encumbrance was obtained from a visual analogue scale (VAS). We adopted an interval scale, which was a $10 \mathrm{~cm}$ horizontal VAS, ranging from 0 (no encumbrance) to 10 (worst imaginable encumbrance) [17]. The subjects had to indicate their bronchial encumbrance perception at the moment of the assessment.

Secondary outcome: Arterial blood gas analysis was obtained from an arterial blood sample taken from the radial artery with the 

exacerbation

patient in resting condition and breathing room air or oxygen (when appropriate) at the prescribed flow rate. Lung function was assessed by means of an automated spirometer: dynamic and static volumes were expressed as \% of their predicted value $[18,19]$. Respiratory muscle strength (maximal inspiratory and expiratory pressure [MIP and MEP]) was then performed by means of a specific module recording maximal pressures against an occlusive mouth resistance at both total lung capacity (for MEP) and functional residual volume (for MIP) [20]. Peak expiratory flow (PEF) and PCEF by a hand-held device was recorded in all patients to confirm their cough competence [14]. Both measures, respiratory muscle strength and cough efficacy, were recorded with the patient in a sitting position; the best of three measurements was recorded. Values were recorded as absolute.

Dyspnea was assessed by the Baseline Dyspnea Index/Transitional Dyspnea Index (BDI/TDI) [21]. Walking capacity was evaluated by means of the distance covered during a 6MWT (6MWD) according to the American Thoracic Society statement [21]. Health status was evaluated using COPD Assessment Test (CAT) and Clinical COPD Questionnaire (CCQ) scores [22].

\section{Statistical analysis}

Analyses were performed with IBM SPSS Statistics 25.0 (Armonk, New York, USA). A preliminary Shapiro-Wilk test was used to assess normality of variables distribution. Data were then reported as means \pm standard deviation (SD) and as medians (interquartile range) for variables normally and not normally distributed, respectively. Categorical variables were considered as frequency (percentage) and analyzed with the Pearson's chi-squared test $\left(\mathrm{X}^{2}\right)$ or the Fisher exact test. Absolute values and changes in each outcome were compared by means of ANOVA and independent-samples $t$-test. Mann-Whitney $U$ test was applied for non-parametric variables. A multivariate general linear model (mixed-model ANOVA) was applied to calculate the statistical interaction between times (baseline and post-PR) and groups (EFA and PEP). The analysis of all outcomes was performed in the intention-to-treat (ITT) population, obtained with the replacing missing data with expectation-maximization (EM) algorithm [23] and data are presented accordingly. The analysis of the primary outcome was performed also in the per-protocol (PP) population. All results were considered to be statistically significant at a level of $p<0.05$.

\section{Results}

The study flow diagram is shown in figure 1 . Thirty-eight out of the 96 screened patients were excluded due to absence of chronic mucus hypersecretion and 24 due to absence of reduced cough efficiency. Baseline characteristics of the 34 patients considered for the study are reported in table 1 . Twenty out of 34 patients were included for Castelrotto and 14 for Tradate. Seven patients dropped out and then 27 patients completed the study and were considered for the study analysis (14 EFA, $13 \mathrm{PEP}$ ). Chronic respiratory failure needing longterm oxygen therapy occurred in seven patients, without difference between the two groups. The two groups were comparable at baseline (Table 1).

Figure 2 reports the primary study outcome (VAS bronchial encumbrance) in ITT and PP population. In both populations and groups was evident a significant improvement after PR, that however was better in EFA group (mean difference between times and groups -1.5 score and -1.6 score in ITT and PP, respectively).

The secondary study outcomes are summarized in table 2. After $\mathrm{PR}$, symptoms (CAT), health status (CCQ) and exercise capacity
Table 1. General characteristics according to the study treatment.

\begin{tabular}{|c|c|c|c|}
\hline Variables & $\begin{array}{c}\text { EFA group } \\
\qquad N=16\end{array}$ & $\begin{array}{c}\text { PEP group } \\
\quad N=18\end{array}$ & p-value \\
\hline Age, years & $73.3 \pm 4.8$ & $72.3 \pm 8.6$ & 0.690 \\
\hline Male, n (\%) & $9(56)$ & $9(50)$ & 0.716 \\
\hline BMI, $\mathrm{kg} / \mathrm{m}^{2}$ & $23[6.8]$ & $24[6.8]$ & 0.446 \\
\hline Pack/year & $58.2 \pm 17.7$ & $54 \pm 16.2$ & 0.471 \\
\hline Previous AECOPD, $\mathrm{n}$ & $2[1]$ & $2[1]$ & 0.641 \\
\hline mMRC, score & $3[1]$ & $2[1]$ & 0.226 \\
\hline CAT, score & $18 \pm 6.9$ & $22.1 \pm 7.8$ & 0.123 \\
\hline BDI, total score & $4.3 \pm 1.9$ & $4.2 \pm 2.4$ & 0.906 \\
\hline VAS bronchial encumbrance, score & $5[2]$ & $5[2]$ & 0.833 \\
\hline CCQ, score & $2.9 \pm 1.1$ & $2.9 \pm 1.1$ & 0.818 \\
\hline $\mathrm{FEV}_{1}, \%$ predicted & $35.1 \pm 7.6$ & $37.5 \pm 9.7$ & 0.427 \\
\hline $\mathrm{FEV}_{1}, \mathrm{FVC}, \%$ & $42.2 \pm 9.2$ & $45.1 \pm 9$ & 0.348 \\
\hline $\mathrm{RV}, \%$ predicted & $203.5 \pm 42.9$ & $192.5 \pm 29.4$ & 0.388 \\
\hline TLC, $\%$ predicted & $143.4 \pm 29$ & $130.8 \pm 23.1$ & 0.170 \\
\hline $\mathrm{DL}_{\mathrm{CO}}, \%$ predicted & $45.4 \pm 8.8$ & $43.9 \pm 9.5$ & 0.653 \\
\hline MIP, $\mathrm{cmH}_{2} \mathrm{O}$ & $53.9 \pm 14.3$ & $47.1 \pm 11.8$ & 0.133 \\
\hline $\mathrm{MEP}, \mathrm{cmH}_{2} \mathrm{O}$ & $78.7 \pm 20.6$ & $72.6 \pm 14$ & 0.318 \\
\hline PEF, L/m & $175[25]$ & $162.5[92.5]$ & 0.395 \\
\hline PCEF, L/m & $180[47.5]$ & $160[27.5]$ & 0.187 \\
\hline $\mathrm{PaO}_{2}, \mathrm{mmHg}$ & $67.6 \pm 9.5$ & $66 \pm 8.6$ & 0.604 \\
\hline $\mathrm{PaCO}_{2}, \mathrm{mmHg}$ & $45.1 \pm 6.7$ & $45.4 \pm 9.9$ & 0.914 \\
\hline 6MWD, meters & $302.7 \pm 115$ & $296.3 \pm 97.1$ & 0.861 \\
\hline Drop-out, n (\%) & $2(12)$ & $5(28)$ & 0.405 \\
\hline
\end{tabular}

Data are shown as number of subjects (\%), means \pm SD or medians [interquartile range], unless otherwise stated.

Abbreviations: EFA indicates Expiratory Flow Accelerator technique; PEP, positive expiratory pressure; BMI, body mass index; AECOPD, acute exacerbation of COPD; mMRC, modified Medical Research Council dyspnoea score; CAT, COPD assessment test; BDI, baseline dyspnea index; VAS, visual analogic scale; CCQ, COPD questionnaire score; FEV , forced expiratory volume at $1^{\text {st }}$ second; FVC, forced vital capacity; RV, residual volume; TLC, total lung capacity; $\mathrm{DL}_{\mathrm{CO}}$, diffusion capacity for carbon monoxide; MIP and $\mathrm{MEP}$, maximal inspiratory and expiratory pressure respectively; $\mathrm{PEF}$, peak expiratory flow; PCEF, peak cough expiratory flow; $\mathrm{PaO}_{2}$ partial arterial oxygen pressure; $\mathrm{PaCO}_{2}$, partial arterial carbon dioxide pressure; $6 \mathrm{MWD}$, six-minute walking distance.

(6MWD) significantly improved in both groups $(\mathrm{p}<0.05)$. Static $(\mathrm{RV}-$ residual volume) and dynamic volumes (FVC-forced vital capacity) significantly improved in both groups, while total lung capacity (TLC) and MIP only in the EFA group $(\mathrm{p}<0.05)$. No significant changes were recorded for $\mathrm{FEV}_{1}$, TDI, MEP, PEF, PCEF and arterial blood gases variables.

\section{Discussion}

In this study, we investigated the value of EFA technique in facilitating airways clearing in COPD patients with severe airflow obstruction, chronic mucus hypersecretion and reduced cough efficiency, after severe exacerbation. The main result of this study was that EFA may enhance airway clearance in these patients, improving lung volumes and inspiratory muscle strength and thus reducing the perceived bronchial encumbrance.

Specific recommendations in international guidelines regarding ACTs for patients with AECOPD are not common [2]. This is likely due to the limited evidence of clinical benefit that has been previously reported [4]. This review reported a statistically significant pooled benefit for ACTs (compared to usual care) in reducing the need for or duration of assisted ventilation during AECOPD, with larger effects suggested for PEP-based versus non-PEP-based techniques [4]. A subsequent large randomized controlled trial of PEP therapy for patients with AECOPD, however, failed to show any appreciable benefit on a range of clinically important outcomes [5]. 
Table 2. Secondary study outcomes.

\begin{tabular}{|c|c|c|c|c|c|c|c|c|}
\hline \multirow[b]{2}{*}{ Variables } & \multicolumn{3}{|c|}{ EFA group } & \multicolumn{3}{|c|}{ PEP group } & \multirow[t]{2}{*}{$\begin{array}{c}\text { Mean difference } \\
(95 \% \text { CI })\end{array}$} & \multirow[t]{2}{*}{ p-value } \\
\hline & Baseline & Post-PR & p-value & Baseline & Post-PR & p-value & & \\
\hline CAT, score & $18 \pm 6.9$ & $10.9 \pm 4.1$ & 0.001 & $22.1 \pm 7.8$ & $12.8 \pm 4.6$ & $<0.001$ & $2.1(-2.4$ to 6.5 & 0.351 \\
\hline BDI/TDI, total score & $4.3 \pm 1.9$ & $3.5 \pm 2.3$ & 0.295 & $4.2 \pm 2.4$ & $3.2 \pm 1.2$ & 0.079 & $0.2(-1.7$ to 2.1$)$ & 0.821 \\
\hline CCQ, score & $2.9 \pm 1.1$ & $1.8 \pm 0.3$ & $<0.001$ & $2.9 \pm 1.1$ & $1.7 \pm 0.6$ & $<0.001$ & $-0.01(-0.57$ to 0.61$)$ & 0.946 \\
\hline $\mathrm{FEV}_{1}, \%$ predicted & $35.1 \pm 7.6$ & $36.1 \pm 6.4$ & 0.583 & $37.5 \pm 9.7$ & $40.4 \pm 11.3$ & 0.116 & $-1.8(-7.1$ to 3.5$)$ & 0.487 \\
\hline FVC, \% predicted & $66.1 \pm 14$ & $74.9 \pm 14.2$ & 0.035 & $64.2 \pm 16.7$ & $70.1 \pm 14.8$ & 0.040 & $2.8(-6.4$ to 12.1$)$ & 0.532 \\
\hline RV, \% predicted & $203.5 \pm 42.9$ & $170.6 \pm 36.0$ & 0.010 & $192.5 \pm 29.4$ & $174.3 \pm 23.0$ & 0.037 & $-14.6(-42.3$ to 13$)$ & 0.290 \\
\hline TLC, $\%$ predicted & $143.4 \pm 29$ & $125.3 \pm 15.4$ & 0.018 & $130.8 \pm 23.1$ & $120.6 \pm 14.7$ & 0.082 & $-7.8(-25.7$ to 10$)$ & 0.378 \\
\hline MIP & $53.9 \pm 14.3$ & $61 \pm 17.3$ & 0.009 & $47.1 \pm 11.8$ & $47.1 \pm 8$ & 0.976 & $7(0.2$ to 13.9$)$ & 0.043 \\
\hline MEP & $78.7 \pm 20.6$ & $78.8 \pm 20.7$ & 0.949 & $72.6 \pm 14$ & $75.8 \pm 13.8$ & 0.449 & $-3.1(-13.5$ to 7.3$)$ & 0.551 \\
\hline PEF & $174.4 \pm 58.3$ & $183.6 \pm 38.7$ & 0.188 & $159.1 \pm 54.4$ & $170.3 \pm 35.3$ & 0.192 & $-1.9(-23.9$ to 20$)$ & 0.857 \\
\hline PCEF & $190.6 \pm 40.2$ & $204.6 \pm 21.7$ & 0.079 & $175.5 \pm 41.3$ & $190 \pm 26.4$ & 0.127 & $-0.4(-24.6$ to 23.7$)$ & 0.970 \\
\hline $\mathrm{PaO}_{2}, \mathrm{mmHg}$ & $67.6 \pm 9.5$ & $70.4 \pm 8.6$ & 0.253 & $66 \pm 8.6$ & $68.6 \pm 6.3$ & 0.159 & $0.2(-5.7$ to 6$)$ & 0.946 \\
\hline $\mathrm{PaCO}_{2}, \mathrm{mmHg}$ & $45.1 \pm 6.7$ & $44.3 \pm 3.9$ & 0.434 & $45.4 \pm 9.9$ & $43.9 \pm 6.3$ & 0.273 & $0.7(-2.8$ to 4.2$)$ & 0.694 \\
\hline 6MWD, meters & $302.7 \pm 115$ & $364.5 \pm 90.4$ & 0.003 & $296.3 \pm 97.1$ & $355.6 \pm 89.5$ & $<0.001$ & $2.5(-37.3$ to 42.3$)$ & 0.894 \\
\hline
\end{tabular}

Data are shown as means $\pm \mathrm{SD}$.

Abbreviations: TDI, transitional dyspnea index. For the other abbreviations see Table 1.

COPD patients with a recent severe AECOPD admitted to a pulmonary rehabilitation program in the period between June 2017 and December 2018 $(N=96)$

Patients excluded $(N=62)$ due to:

- Absence of chronic mucus hypersecretion $(N=38)$

- Absence of reduced cough efficiency $(N=24)$

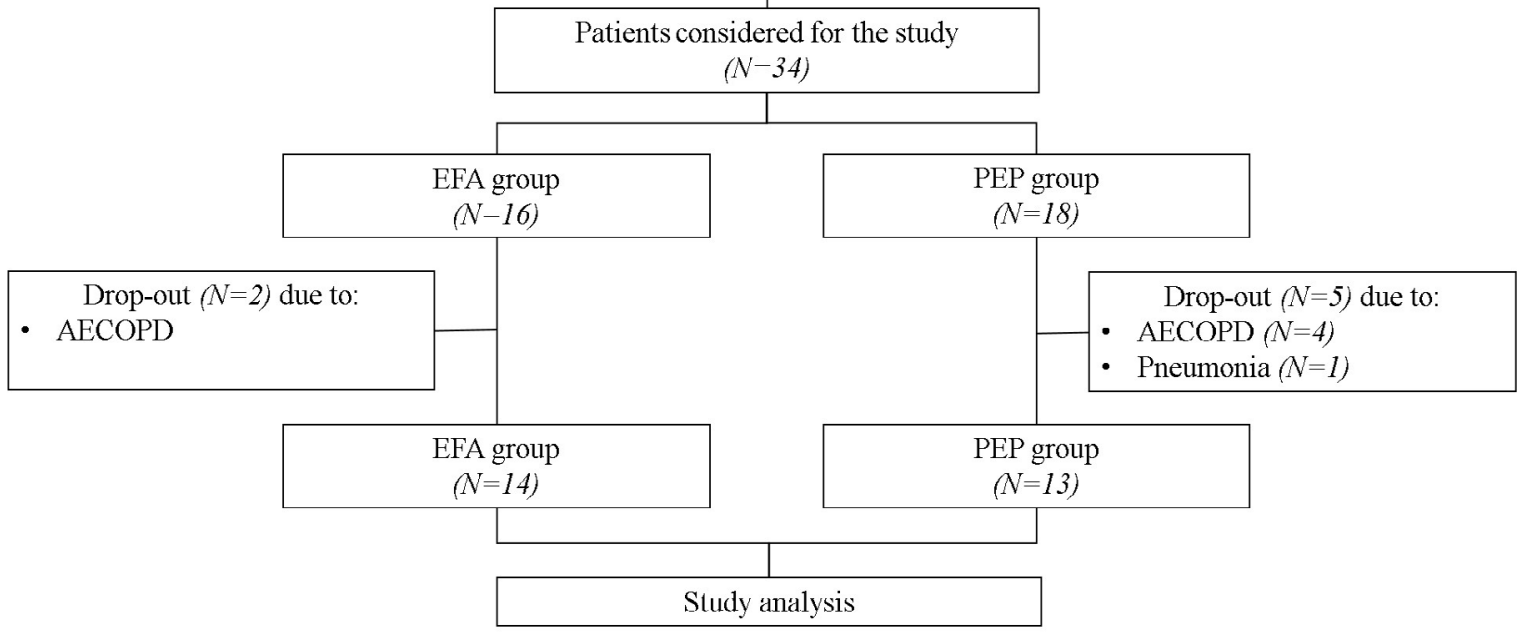

Figure 1. Study flow diagram

Abbreviations: PR indicates pulmonary rehabilitation; EFA, expiratory flow accelerator; PEP, positive expiratory pressure; AECOPD, acute exacerbation of chronic obstructive pulmonary disease.

Many different types of ACTs exist such as breathing exercises, positioning and use of adjunct devices such as PEP therapy or mechanical chest wall oscillation [24]. Choice of technique is most commonly determined by respiratory therapists in close consideration of patients' individual needs. The lack of standardized recommendations in this field has certainly contributed to the well-documented variability of treatment regimens within and between countries [25-27].

Up to now, there are very few studies of EFA technology and most of these are congress communications [9-12]. To the best of our knowledge, this is the first study to evaluate the effect of EFA technology on airway clearance in COPD patients after acute exacerbations. Our preliminary study compared EFA technology to PEP-bottle, a widely used PEP technique in severe to very severe COPD patients. There is only one previous report that considered a similar population [12]. However, there are a number of differences between our study and this previous report. Patrizio et al. enrolled severe COPD subjects in stable conditions [12]. Moreover, in our study we selected patients with reduced cough efficiency. On the other hand, the presence of hypersecretion and the sample size were similar in the two studies [12]. Differences between the two-study population may explain the differences in the results observed. Whereas Patrizio et al. showed 

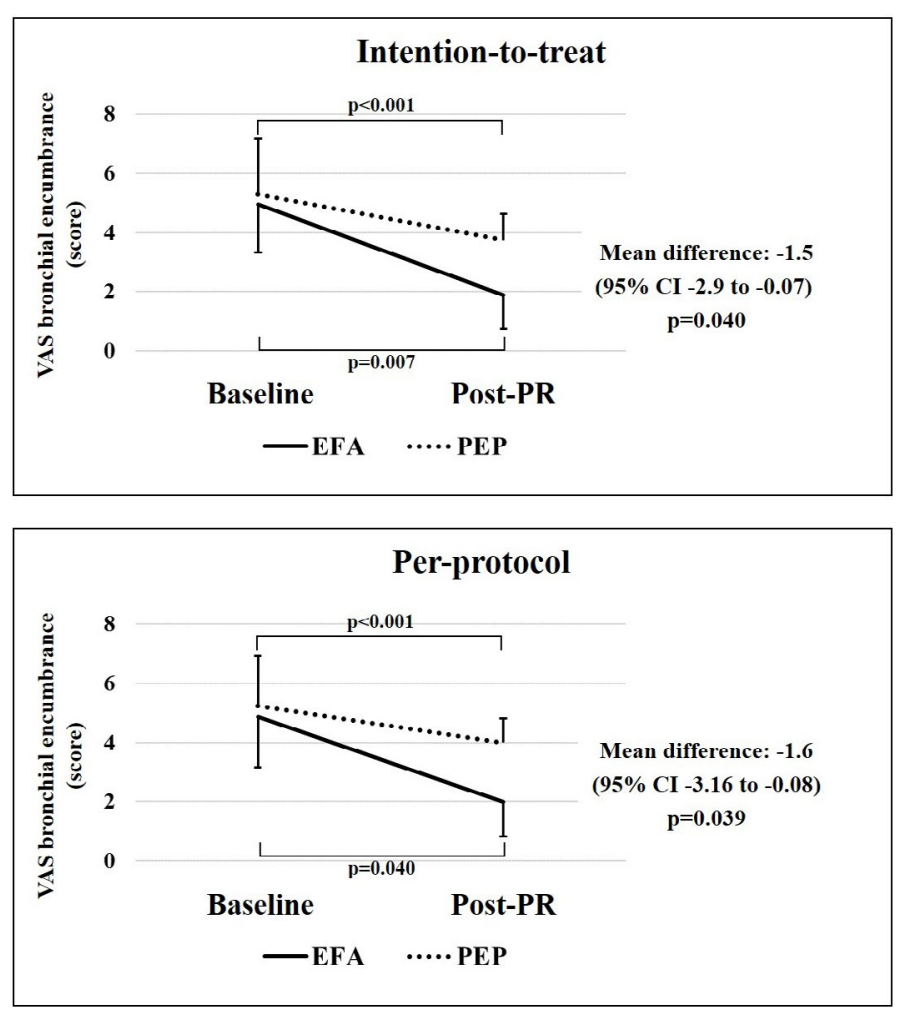

Figure 2. VAS bronchial encumbrance evaluated at baseline and post-PR in intention-totreat and per-protocol populations.

Abbreviations: VAS indicates visual analogue scale of bronchial encumbrance; PR, pulmonary rehabilitation; EFA, expiratory flow accelerator; PEP, positive expiratory pressure.

an improvement in gas exchanges for EFA, in the present study, we observed an improvement in perceived bronchial encumbrance in both groups, with a greater magnitude in the study group. Interestingly, two previous studies evaluated the use of EFA in pediatric subjects with ineffective cough $[9,10]$. Although with different nuances, both studies observed a reduction in respiratory problems, assessed as number of respiratory exacerbations, number of hospital admissions, days spent in hospital, and home visits and pharmacological therapy prescribed by primary care $[9,10]$. Based on these data, we can speculate that EFA technology may provide a greater contribution to airway clearance, and particularly in patients with reduced cough efficiency.

In addition, we found an improvement in lung volumes (FVC, TLC and RV) and in MIP in EFA group, which is likely to prove both the reduction in airway obstruction and the recruitment of collapsed or obstructed peripheral airways and lung parenchyma. The theoretical benefit of these techniques, indeed, is the ability to enhance mucus clearance by either stenting the airways and preventing airway collapse, or increasing intrathoracic pressure and collateral ventilation distal to retained secretions, or decreasing functional residual capacity of the lung [28].

Finally, we also observed an improvement in health status in both groups. Previous studies using physiotherapy techniques showed discordant results [29-31]. The study of Nicolini [29] and Kodric [30] showed positive results regarding modified Medical Research Council dyspnoea score (mMRC), CAT and Borg scores, whereas the large trial of Cross [31] found no difference in quality of life. It is worth noting that the trial had broad inclusion criteria and participants did not have to be productive of sputum to take part [31].
Despite reporting some original finding with a potential clinical significance, this study has some limitations. Firstly, the small sample size might limit the validity of the results observed. The considerable selection of patients forced us to exclude most of the patients evaluated. On the other hand, identifying a COPD responder to ACT group remains a potentially valuable clinical prospect, however, this may prove unfeasible due to the significant sample sizes required for statistical power when using outcomes of high clinical importance. Similarly, no inferences can be made about the maintenance of these results in the long term, due to the short duration of the study.

\section{Conclusion}

In conclusion, we evaluated the efficacy of EFA in improving the bronchial clearance in COPD patients with hypersecretion and reduced cough efficiency after severe exacerbation, in comparison with PEP-bottle. We observed a potential benefit with EFA technique in these patients. As an exploratory study, further studies are needed to confirm our results.

\section{Conflict of interest statements}

The authors declare that the publication fee was paid by Medical Products Research Srl Italy.

\section{References}

1. Global Initiative for Chronic Obstructive Lung Disease (2019) Global Strategy for the Diagnosis, Management, and Prevention of Chronic Obstructive Pulmonary Disease. Date last accessed: March 6, 2019.

2. Wedzicha JA, Miravitlles M, Hurst JR, Calverley PM, Albert RK, et al. (2017) Management of COPD exacerbations: a European Respiratory Society/American Thoracic Society guideline. Eur Respir J 49(3):1600791. [Crossref]

3. Holland AE, Button BM (2006) Is there a role for airway clearance techniques in chronic obstructive pulmonary disease? Chron Respir Dis 3: 83-91. [Crossref]

4. Osadnik CR, McDonald CF, Jones AP, Holland AE (2012) Airway clearance techniques for chronic obstructive pulmonary disease. Cochrane Database Syst Rev 3: CD008328. [Crossref]

5. Osadnik CR, McDonald CF, Miller BR, Hill CJ, Tarrant B, et al. (2014) The effect of positive expiratory pressure (PEP) therapy on symptoms, quality of life and incidence of re-exacerbation in patients with acute exacerbations of chronic obstructive pulmonary disease: a multicentre, randomised controlled trial. Thorax 69: 137-143. [Crossref]

6. Hill K, Patman S, Brooks D (2010) Effect of airway clearance techniques in patients experiencing an acute exacerbation of chronic obstructive pulmonary disease: a systematic review. Chron Respir Dis 7: 9-17. [Crossref]

7. Kim CS, Rodriguez CR, Eldridge MA, Sackner MA (1986) Criteria for mucus transport in the airways by two-phase gas-liquid flow mechanism. J Appl Physiol 60: 901-907. [Crossref]

8. Kim CS, Iglesias AJ, Sackner MA (1987) Mucus clearance by two phase gas-liquid flow mechanism: asymmetric periodic flow model. J Appl Physiol 62: 959-971. [Crossref]

9. Garuti G, Verucchi E, Fanelli I, Giovannini M, Winck JC, et al. (2016) Management of bronchial secretions with Free Aspire in children with cerebral palsy: impact on clinical outcomes and healthcare resources. Ital J Pediatr 42: 7. [Crossref]

10. Bertelli L, Bardasi G, Cazzato S, Di Palmo E, Gallucci M, et al. (2019) Airway clearance management with vaküm technology in subjects with ineffective cough: a pilot study on the efficacy, acceptability evaluation, and perception in children with cerebral palsy. Pediatr Allergy Immunol Pulmonol 32: 23-27. [Crossref]

11. Bertelli, L, Di Nardo G, Cazzato S, Ricci G, Pession A (2017) Free-Aspire: A new device for the management of airways clearance in patient with ineffective cough. Pediatr Rep 9: 7270. [Crossref]

12. Patrizio G, D'Andria M, D'Abrosca F, et al. (2018) Airway clearance with expiratory flow accelerator technology: effectiveness of the "free aspire" device in patients with severe COPD. Turk Thorac J 30: 209-215. [Crossref]

13. Bott J, Blumenthal S, Buxton M, Ellum S, Falconer C, et al. (2009) on behalf of the British Thoracic Society Physiotherapy Guideline Development Group. Guidelines for the physiotherapy management of the adult, medical, spontaneously breathing patient Thorax 64: i1-i52. [Crossref] 
14. Chatwin M, Ross E, Hart N, Nickol AH, Polkey MI, et al. (2003) Cough augmentation with mechanical insufflation/exsufflation in patients with neuromuscular weakness. Eur Respir J 21: 502-508. [Crossref]

15. Spruit MA, Singh SJ, Garvey C, ZuWallack R, Nici L, et al. (2013) An official American Thoracic Society/European Respiratory Society statement: key concepts and advances in pulmonary rehabilitation. Am J Respir Crit Care Med 188: e13-e64. [Crossref]

16. Zainuldin MR, Knoke D, Mackey MG, Luxton N, Alison JA (2007) Prescribing cycle training intensity from the six-minute walk test for patients with COPD. BMC Pulm Med 7: 9. [Crossref]

17. Aitken RC (1969) Measurement of feelings using Visual Analogue Scales. Proc R Soc Med 62: 989-993. [Crossref]

18. Quanjer PH, Tammeling GJ, Cotes JE, Pedersen OF, Peslin R, et al. (1993) Lung volumes and forced ventilatory flows. Report Working Party Standardization of Lung Function Tests, European Community for Steel and Coal. Official Statement of the European Respiratory Society. Eur Respir J Suppl 16: 5-40. [Crossref]

19. Cotes JE, Chinn DJ, Quanjer PH, Roca J, Yernault JC (1993) Standardization of the measurement of transfer factor (diffusing capacity). Report Working Party Standardization of Lung Function Tests, European Community for Steel and Coal. Official Statement of the European Respiratory Society. Eur Respir J Suppl 16: 41-52. [Crossref]

20. Bruschi C, Cerveri I, Zoia MC, Fanfulla F, Fiorentini M, et al. (1992) Reference values of maximal respiratory mouth pressures: a population-based study. Am Rev Respir Dis 146 (Suppl. 3): 790-793. [Crossref]

21. Mahler DA, Guyatt GH, Jones PW (1998) Clinical measurements of dyspnea; in Mahler DA (ed): Dyspnea. New York, Marcel Dekker. pp: 149-198.

22. ATS Committee on Proficiency Standards for Clinical Pulmonary Function Laboratories (2002) ATS statement: guidelines for the six-minute walk test. Am J Respir Crit Care Med 166: 111-117. [Crossref]
23. Dempster AP, Laird NM, Rubin DB (1977) Maximum likelihood from incomplete data via the EM algorithm. J Roy Stat Soc Series B 39: 1-38

24. Fagevik Olsén M, Lannefors L, Westerdahl E (2015) Positive expiratory pressure Common clinical applications and physiological effects. Respir Med 109: 297-307. [Crossref]

25. Yohannes AM, Connolly MJ (2007) A national survey: percussion, vibration, shaking and active cycle breathing techniques used in patients with acute exacerbations of chronic obstructive pulmonary disease. Physiotherapy 93: 110-113.

26. Harth L, Stuart J, Montgomery C, Pintier K, Czyzo S, et al. (2009) Physical therapy practice patterns in acute exacerbations of chronic obstructive pulmonary disease. Can Respir J 16: 86-92. [Crossref]

27. Osadnik CR, McDonald CF, Holland AE (2013) Airway clearance techniques in acute exacerbations of COPD: a survey of Australian physiotherapy practice. Physiotherapy 99: 101-106. [Crossref]

28. Nunn JF. Nunn's Applied Respiratory Physiology, 5th ed. Oxford: ButterworthHeinemann, 2000.

29. Nicolini A, Mascardi V, Grecchi B, Ferrari-Bravo M, Banfi P, et al. (2018) Comparison of effectiveness of temporary positive expiratory pressure versus oscillatory positive expiratory pressure in severe COPD patients. Clin Respir J 12: 1274-1282. [Crossref]

30. Kodric M, Garuti G, Colomban M, Russi B, Porta RD, et al. (2009) The effectivenes of a bronchial drainage technique (ELTGOL) in COPD exacerbations. Respirology 14: 424-428. [Crossref]

31. Cross J, Elender F, Barton G, Clark A, Shepstone L, et al. (2010) A randomized controlled equivalence trial to determine the effectiveness and cost-utility of manual chest physiotherapy techniques in the management of exacerbations of chronic obstructive pulmonary disease (MATREX). Health Technol Assess 14: 1-147. [Crossref]

Copyright: (C2019 Zampogna E. This is an open-access article distributed under the terms of the Creative Commons Attribution License, which permits unrestricted use, distribution, and reproduction in any medium, provided the original author and source are credited. 